\title{
Persistent Cocaine-Induced Reversal Learning Deficits Are Associated with Altered Limbic Cortico-Striatal Local Field Potential Synchronization
}

\author{
Clinton B. McCracken and Anthony A. Grace \\ Departments of Neuroscience, Psychiatry, and Psychology, University of Pittsburgh, Pittsburgh, Pennsylvania 15260
}

\begin{abstract}
Repeated exposure to cocaine is known to produce persistent deficits in behavioral flexibility. Evidence suggests that these deficits are mediated in part by a circuit involving the medial prefrontal and orbitofrontal cortices (PFC and OFC), nucleus accumbens (NAC), and basolateral amygdala (BLA). To assess the effects of cocaine on this circuit, we treated rats with cocaine daily for $14 \mathrm{~d}$, followed by 4 weeks of abstinence. Animals were then tested on a cross-maze-based reversal learning and set-shifting task, after which they were anesthetized to allow for recording of spontaneous local field potential (LFP) activity simultaneously from all four regions, in addition to activity evoked from acute BLA stimulation. Cocaine-treated (COC) animals showed specific deficits in reversal learning; furthermore, spontaneous LFP oscillation power was reduced and BLA-induced oscillation power was increased in all regions compared with saline-treated (SAL) rats. Theta-burst stimulation of BLA potentiated BLA-evoked responses in all regions and cocaine challenge reduced spontaneous oscillation power and evoked response amplitude, with no COC/SAL group differences. Notably, cocaine challenge produced differential changes in coherence between OFC-BLA, BLA-NAC, and OFC-NAC in COC and SAL groups. These data indicate that repeated exposure to cocaine can produce changes in oscillatory LFP synchronization along limbic cortico-striatal circuits that persist long into abstinence. Furthermore, the regional specificity of these changes strongly correlates with the observed behavioral deficits. Aberrant synchronization within and between regions and consequent dysregulation of the neurocircuitry involved in executive control may contribute to the long-lasting maladaptive decision making seen in cocaine abusers.
\end{abstract}

\section{Introduction}

Cocaine addiction is characterized by persistent drug-taking despite adverse consequences (Mendelson and Mello, 1996), with addicts exhibiting aberrant decision making with respect to inhibitory control of behavior and flexible switching between strategies (Jentsch and Taylor, 1999; Robbins and Everitt, 1999; Rogers and Robbins, 2001). Humans and animals exposed to cocaine show drastic deficits in reversal learning, wherein associations between cues or actions and outcomes must be reversed (Jentsch et al., 2002; Schoenbaum et al., 2004; Fillmore and Rush, 2006; Calu et al., 2007; Ersche et al., 2008). While cocaineinduced deficits in reversal learning are well characterized, effects on set-shifting in humans remain equivocal. Although several studies report that cocaine users are impaired in these tasks (Ardila et al., 1991; Colzato et al., 2009; Hanlon et al., 2011; Soar et

\footnotetext{
Received April 4, 2013; revised Sept. 17, 2013; accepted Sept. 29, 2013.

Author contributions: C.B.M. designed research; C.B.M. performed research; C.B.M. analyzed data; C.B.M. and A.A.G. wrote the paper.

This work was supported by National Institutes of Health grants MH57440, DA15408, and MH45156 (to A.A.G.) and a National Alliance for Research on Schizophrenia and Depression Young Investigator Award and Tourette Syndrome Association Fellowship (to C.B.M.). We thank Nicole Macmurdo and Grace Lindsay for technical assistance. The authors declare no competing financial interests.

Correspondence should be addressed to Clinton B. McCracken, Departments of Neuroscience, Psychiatry, and Psychology, A210 Langley Hall, University of Pittsburgh, Pittsburgh, PA 15260. E-mail: clinton. mccracken1@gmail.com.

C.B. McCracken's present address: Department of Clinical Neurosciences and Hotchkiss Brain Institute, University of Calgary, Calgary, AB T2N 4N1, Canada.

DOI:10.1523/JNEUROSCI.1440-13.2013

Copyright $\odot 2013$ the authors $\quad 0270-6474 / 13 / 3317469-14 \$ 15.00 / 0$
}

al., 2012), others suggest they perform no worse than controls (Gillen et al., 1998; Bolla et al., 1999; Goldstein et al., 2004; Madoz-Gúrpide et al., 2011). In animals, prenatal cocaine exposure impaired shifting from an olfactory to a spatial cue but not the converse (Garavan et al., 2000), and cocaine-treated animals reached criterion as fast as controls but made more perseverative errors (Goto and Grace, 2005).

These cognitive deficits are thought to rely on altered limbic and frontostriatal interactions. The prefrontal cortex (PFC) is heavily implicated in behavioral flexibility. In rats, damage or inactivation of the orbitofrontal cortex (OFC) is associated with impaired reversal learning but not set-shifting, and the opposite is true with medial prefrontal cortex (mPFC; Ragozzino et al., 1999; Birrell and Brown, 2000; Chudasama and Robbins, 2003; McAlonan and Brown, 2003; Floresco et al., 2006b; Boulougouris et al., 2007; Ghods-Sharifi et al., 2008). Both of these regions have reciprocal connections with the basolateral amygdala (BLA; Krettek and Price, 1977; Cassell and Wright, 1986; McDonald, 1987, 1991; Cassell et al., 1989; Mcdonald et al., 1996), which itself encodes aspects of cue-outcome associations and exhibits cocaine-induced alterations in cue responsivity (Childress et al., 1999; Bonson et al., 2002; Carelli et al., 2003; Stalnaker et al., 2007b). Projections from OFC, PFC, and BLA converge on the nucleus accumbens (NAC; Finch, 1996; Mcdonald, 1996; Groenewegen et al., 1999), leaving it well positioned to integrate information from limbic, motor, and cognitive regions (Mogenson et al., 1980; Groenewegen and Trimble, 2007). 
Although a number of studies have characterized various cocaine-induced neuroadaptations in these areas, scant data have been reported regarding persistent changes with respect to synchronization of activity within and between regions along this circuit. We exposed rats to a cocaine regimen shown to produce enduring deficits in reversal learning (Schoenbaum et al., 2004; Stalnaker et al., 2006). After 4 weeks of withdrawal, animals were tested on the acquisition, retention, and reversal of an egocentric response, followed by a set-shift. We then anesthetized the animals and recorded local field potential (LFP) activity simultaneously from OFC, PFC, NAC, and BLA, probed differences in the effects of theta-burst stimulation on BLA-evoked responses, and examined the effects of an intravenous cocaine challenge on spontaneous and evoked LFP activity.

\section{Materials and Methods}

All procedures were performed in accordance with the guidelines outlined in the National Institutes of Health (NIH) Guide for the Care and Use of Laboratory Animals, and were approved by the Institutional Animal Care and Use Committee of the University of Pittsburgh.

Animals and locomotor behavior. Male Sprague Dawley rats (275-400 g; Harlan) were housed on a reverse light/dark cycle (lights on 7:00 P.M. to 7:00 A.M.) for at least 2 weeks and handled for 5-10 min daily for $5 \mathrm{~d}$ before the behavioral experiments. A time line for the behavioral experiments is presented in Figure 1. Animals were habituated to the locomotor chambers (Coulbourn Instruments) where spontaneous locomotor activity in the horizontal plane and stereotyped movements were monitored by photobeam breaks and recorded using TruScan software (Coulbourn Instruments). Animals were habituated to the locomotor chambers for 60 min sessions on 2 consecutive days. Rats were then divided into saline (SAL) and cocaine (COC) treatment groups, counterbalanced according to locomotor activity during habituation sessions. Beginning the day after the last habituation session, locomotion and stereotypy were assessed after animals received daily injections of either saline $(1 \mathrm{ml} / \mathrm{kg}$, i.p.) or cocaine $(30 \mathrm{mg} / \mathrm{kg}$, i.p.). After collecting $30 \mathrm{~min}$ of baseline activity, animals were injected and activity monitored for a further $90 \mathrm{~min}$.

\section{Cross-maze task}

Maze setup. The maze was constructed from Plexiglas $(0.63 \mathrm{~cm}$ thick) painted opaque gray, and consisted of four arms connected to a $14 \mathrm{~cm}^{2}$ central area. The arms were $14 \mathrm{~cm}$ wide, $50 \mathrm{~cm}$ long, and $25 \mathrm{~cm}$ high. A food well $1.9 \mathrm{~cm}$ in diameter and $0.75 \mathrm{~cm}$ deep was located $\sim 2.5 \mathrm{~cm}$ from the distal end of each arm. The food well was sufficiently deep to hide the food pellet from the view of the rat from the arm entrance. Removable pieces of gray opaque Plexiglas $(20 \times 10 \mathrm{~cm})$ were used to block the arms of the maze to form a "T" configuration. The holding cage used for intertrial intervals was constructed of gray-painted Plexiglas and measured $35 \times 35 \times 35 \mathrm{~cm}$.

Habituation. The habituation procedure used here was adapted from prior studies (Ragozzino, 2002; Floresco et al., 2006a). Briefly, on the 3 consecutive days before initial exposure to the maze, rats were given $\sim 20$ sweetened food reward pellets (Bio-Serv) in a plastic dish in the home cage, which was removed once all of the pellets were eaten. On day one of habituation, five reward pellets were placed in each of the arms of the maze (three down the length of the arm and two in the food well). A rat was placed in the maze and allowed to explore and consume the food pellets ad libitum for $15 \mathrm{~min}$. If the rat consumed all pellets within $15 \mathrm{~min}$, it was removed from the maze and placed in the holding cage. The maze was then rebaited with 12 additional pellets, and the rat was placed back in the center of the maze. On day two, arms were only baited with three pellets each (two in the food wells and one in the center of the arms). Whenever a rat traversed the entire length of an arm and consumed the two food pellets in the well, it was picked up and placed at the entrance of a different arm. This procedure was performed to habituate the animal to repeated handling immediately after consuming food reward. Subsequent habituation sessions were similar to the second day, except that only one food pellet was placed at the end of each arm. This procedure was then repeated daily until the rat had consumed all four food pellets on the maze at least four times in the $15 \mathrm{~min}$ period. All rats reached this criterion within $7 \mathrm{~d}$.

After maze habituation, we next determined the rat's turn bias. The opaque Plexiglas insert was placed at the entrance of one of the arms to form $\mathrm{T}$ configuration. A rat was placed in the stem arm and allowed to turn left or right to obtain a food pellet. In one of the choice arms, a white laminated piece of poster board $(13 \times 30 \mathrm{~cm})$ serving as a visual cue was placed on the floor. After a rat chose an arm and consumed the reward, it was placed in the stem arm, and allowed to make another choice. If the rat chose the same arm as the initial choice, it was returned to the stem arm until it the other arm was chosen. After both arms had been chosen, the rat was returned to the holding cage, the Plexiglas barrier and visual cue were moved to different arms, and a new trial commenced. Thus, a trial for the turn-bias procedure consisted of entering both choice arms and consuming the food pellets. The turn that a rat made first during the initial choice of a trial was recorded and counted toward its turn bias, and the direction (right or left) that a rat turned four or more times over seven trials was considered its turn bias. Testing commenced the following day.

Day 1: response discrimination training. For the initial response discrimination, the animal was required to turn in the opposite direction of its turn bias (left or right), regardless of the location of the visual cue placed in one of the arms. The start arm varied to discourage animals from using an allocentric spatial strategy to locate the food. On day 1 of training, a rat was started from the arms designated West, South, and East. The location of these arms relative to the spatial cues in the room was varied across animals. For each trial, the visual cue was placed in one of the arms so that over every set of 12 trials it appeared equally in each $\mathrm{arm}$. The start locations for each trial and position of the visual cue were determined pseudorandomly. After successfully completing a trial, the rats were placed in a holding cage, with an intertribal interval $\sim 15 \mathrm{~s}$. A rat continued to perform the task until it reached a criterion of 10 correct consecutive choices. There was no maximum limit on the number of trials a rat was allowed to reach this criterion. Following the tenth consecutive correct choice, it received a probe trial involving starting the rat from the fourth arm (North) that was unused during testing. During probe trials, the visual cue was inserted in the arm opposite to the direction that the rat was required to turn. If a rat made a correct response, training was completed. If a rat made an incorrect response, training continued until a rat made an additional five consecutive correct choices, at which time another probe trial was administered. This continued until a rat made a correct choice on the probe trial. For analysis, we examined trials to criterion, defined as the total number of test trials completed before a correct choice on the probe trial was made, and probe trials, defined as the total number of probe trials an animal required to get one correct. Exclusion criteria were set such that if a rat did not complete at least 30 trials within the first hour of training, training was discontinued; however, all animals successfully performed the task.

Day 2: retention. The day after reaching criterion on the response version of the task, they were tested on their ability to recall the previous day's training, such that on Day 2 the procedure from Day 1 was repeated.

Day 3: reversal. On the day following retention testing, rats were now required to turn in the opposite direction from the previous $2 \mathrm{~d}$ with all other parameters unchanged.

Day 4: set-shift. The day after reversal testing, rats were required to enter the arm containing the visual cue, the location of which varied pseudorandomly between left and right arms (the cue was placed in each arm with equal frequency every 12 trials). The procedure was otherwise identical to previous days. For probe trials, the visual cue was always placed in the arm opposite to that which the rat had been trained to enter during reversal learning.

Error scoring. Errors during reversal testing (i.e., entries into the incorrect arm) were broken down into "perseverative" and "regressive" errors using a modification of a well characterized procedure (Ragozzino et al., 1999; Ragozzino, 2002; Floresco et al., 2006a, b). Trials were divided into consecutive blocks of four trials each. Perseverative errors were scored when the rat entered the incorrect arm at least two times per block of four trials. Once a rat made less than three errors in a block, all subsequent errors were no longer scored as perseverative errors because the rat was now choosing a different strategy at least half of the time. These errors 
A

Locomotor Activity

Cocaine $(30 \mathrm{mg} / \mathrm{kg}, \mathrm{IP})$

or saline $X 14$ days

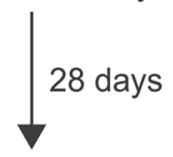

Cross-Maze testing:

Habituation - 3 - 6 days

Response aquisition

Retention

Reversal

4 days

Set-shift

$$
3 \text { - } 5 \text { days }
$$

\section{Electrophysiology}

Record spontaneous and evoked LFP activity, at baseline, following ATBS, and then before and after cocaine ( $3 \mathrm{mg} / \mathrm{kg} \mathrm{IV}$ ) challenge

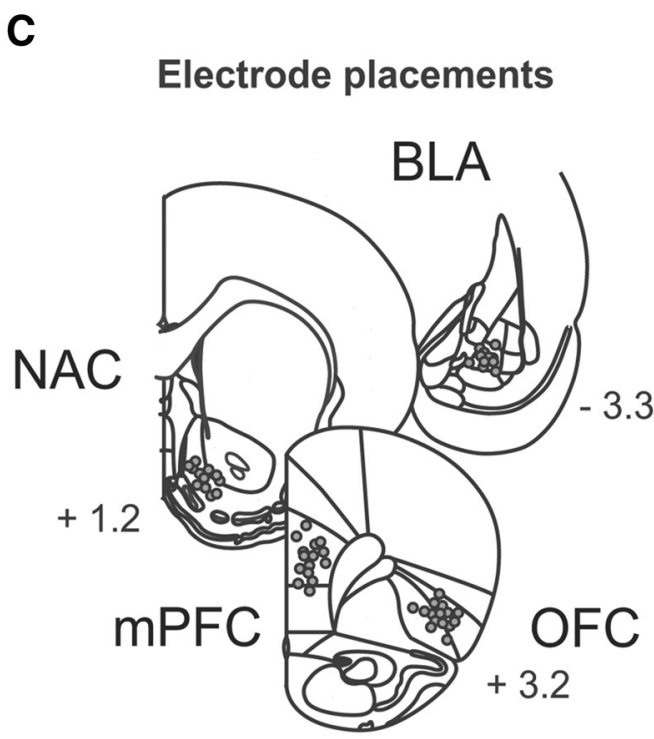

B

Day 1 - Response aquisition
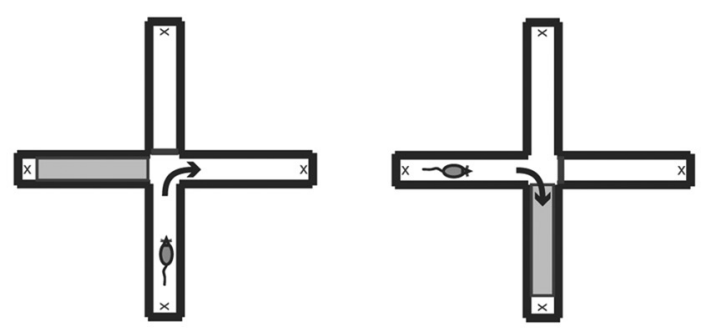

Day 2 - Retention (same as Day 1)

Day 3 - Reversal
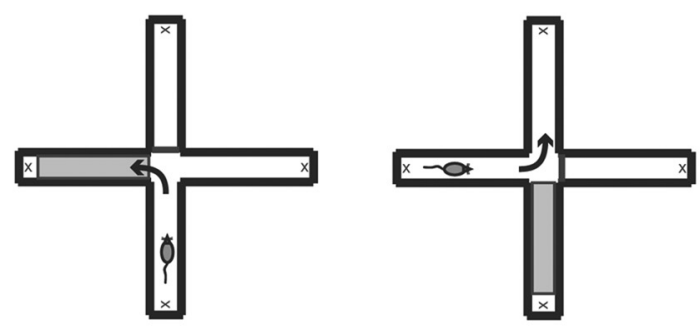

Day 4 - Set shift
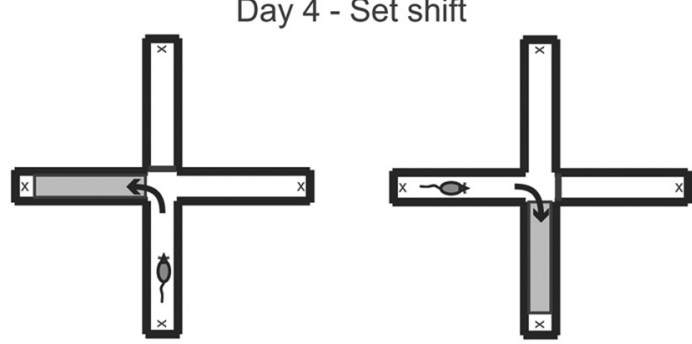

Figure 1. Experimental design. $A$, Outline of protocol. $B$, Schematic of cross-maze task to measure reversal and set-shifting. $C$, Electrode placements in OFC, $m P F C, N A C$, and BLA.

were instead scored as regressive errors. A similar protocol was used with set-shifting, with a slight variation. In every 12 consecutive trials, 6 trials required the rat to enter the arm opposite to the previous day's response. These trials were then grouped into consecutive blocks of four trials each, and perseverative errors scored when the rat entered the incorrect arm at least three times per block of four trials. Similar to above, once errors were no longer preservative, they were scored as either regressive (i.e., away from cue, previous day's direction) or "Day 1" errors (i.e., away from cue, direction for initial discrimination on Day 1). The purpose of this error breakdown was to determine whether cocaine treatment affected the ability to either shift from the previously learned strategy (perseverative errors) or maintain the new strategy after perseveration had ceased (regressive and "Day 1" errors).

\section{Electrophysiology}

Three to five days following maze testing, animals were anesthetized with urethane $(1.5 \mathrm{~g} / \mathrm{kg}$, i.p.) and placed in a stereotaxic frame. Body temperature was maintained at $37^{\circ} \mathrm{C}$ with a temperature-controlled heating pad. 
In all surgical preparations the scalp was exposed and burr holes were drilled in the skull overlying the lateral OFC, PFC, the NAC shell, and the BLA, and the dura resected. A concentric bipolar stimulating electrode (NEX-100; Kopf) was placed in the BLA anteroposterior (AP) $-3.6 \mathrm{~mm}$ (from bregma), mediolateral (ML) $+5.0 \mathrm{~mm}$, and dorsoventral (DV) $-7.5 \mathrm{~mm}$ (from skull); Teflon-insulated stainless steel recording electrodes $(0.1 \mathrm{~mm}$ diameter; Plastics One) were slowly lowered into the OFC (AP: $+3.2 \mathrm{~mm}, \mathrm{ML}:+3.4 \mathrm{~mm}$, DV: $-5.5 \mathrm{~mm}$ ), PFC (AP: +3.2 $\mathrm{mm}, \mathrm{ML}:+0.7 \mathrm{~mm}, \mathrm{DV}:-4 \mathrm{~mm})$, and NAC (AP: $+1.2 \mathrm{~mm}, \mathrm{ML}:+0.7$ $\mathrm{mm}, \mathrm{DV}:-6.9 \mathrm{~mm})$. Recording commenced no earlier than $30 \mathrm{~min}$ following electrode implantation.

Recording. LFPs were amplified (gain =1000), by a multichannel amplifier (A-M Systems) and displayed on an oscilloscope (Tektronix). The data were digitized at either $1 \mathrm{kHz}$ (for spontaneous data) or $10 \mathrm{kHz}$ (for evoked data) and acquired using custom-designed computer software (NeuroScope) and stored for off-line analysis. A skull screw over the contralateral cerebellum was used as a reference for LFP recordings.

For spontaneous recordings, LFPs were recorded for $2 \mathrm{~min}$ at a number of different time points. One pole of the stimulating electrode was used to record spontaneous BLA LFP activity. The spectral power of LFP oscillations in each region and coherence between regions was analyzed using routines from the Chronux software package (www.chronux.org) for MATLAB (MathWorks). For spontaneous LFP data, recordings were segmented (10 s window); each segment was detrended to remove any slow DC components and padded with zeros to increase frequency resolution. Multitaper spectral power and coherence were calculated for each segment in the following frequency bands: slow/delta $(0.5-4 \mathrm{~Hz})$, theta $(4-12 \mathrm{~Hz})$, beta $(13-30 \mathrm{~Hz})$, low gamma $(30-58 \mathrm{~Hz})$, and high gamma $(62-100 \mathrm{~Hz})$. Data for each frequency band were then averaged over segments.

Evoked responses. Acute BLA stimulation produced a negative-going short-latency peak in OFC, PFC, and NAC termed N1; N1 amplitude was defined as the peak voltage deflection subtracted from the voltage value $0.5 \mathrm{~ms}$ before stimulation. For evoked LFP responses, baseline inputoutput curves were generated using four stimulus intensities $(0.1,0.3$, 0.6 , and $1.0 \mathrm{~mA}, 0.2 \mathrm{~ms}$ pulse duration, 30 stimulation sweeps at $0.4 \mathrm{~Hz}$ for each intensity). LFP data were recorded for $800 \mathrm{~ms}$ prestimulus and poststimulus.

Induced oscillations. Acute BLA stimulation also resulted in induced oscillations (i.e., not time locked to the stimulus) in all afferent regions. To quantify the intensity of these induced oscillations and effects, power and coherence in the $750 \mathrm{~ms}$ following each stimulus for each frequency band (beginning $50 \mathrm{~ms}$ poststimulus to avoid the stimulus artifact and acute evoked response) was normalized to activity in the $750 \mathrm{~ms}$ immediately prestimulus for each sweep, averaged over 30 stimulus sweeps at the $1.0 \mathrm{~mA}$ stimulation intensity. Due to the relatively short timescale, analysis of induced oscillatory activity was restricted to theta, beta, and low and high gamma bands.

BLA theta-burst stimulation. Following assessment of baseline spontaneous and induced/evoked activity, theta-burst stimulation (ATBS; three bouts of $10 \times 100 \mathrm{~Hz}$ bursts with a $200 \mathrm{~ms}$ interburst interval; interbout interval, $1 \mathrm{~min}$, intensity, $1 \mathrm{~mA}$ ) was applied to the BLA. The tetanization protocol has been reported to potentiate BLA-evoked responses in PFC and NAC in vivo (Maroun and Richter-Levin, 2003; Gill and Grace, 2011). LFP activity was reassessed immediately following and $30 \mathrm{~min}$ post ATBS. Activity at the $30 \mathrm{~min}$ time point was used as a baseline to compare the effect of intravenous cocaine challenge (see below).

Intravenous cocaine challenge. Following re-establishment of spontaneous and induced/evoked baselines, a cocaine challenge dose $(3 \mathrm{mg} / \mathrm{kg}$, i.v.; via the lateral tail vein) was administered to all animals. Two minutes following cocaine administration, spontaneous and induced/evoked LFP activity was reassessed.

Histology. At the end of each experiment, small lesions were made at the tip of the electrodes ( $250 \mu \mathrm{A}, 10 \mathrm{~s}$ current pulse) and visualized by adding potassium ferrocyanide during postfixation. Animals were killed with an overdose of urethane and decapitated. The brain was removed and fixed for at least $48 \mathrm{~h}$ in $8 \% \mathrm{w} / \mathrm{v}$ paraformaldehyde (in PBS) and cryoprotected in $25 \% \mathrm{w} / \mathrm{v}$ sucrose (in PBS). Brains were then sectioned (50 $\mu \mathrm{m}$ coronal sections), placed on gelatin-chromalum-coated slides, and stained with cresyl violet for histochemical verification of the recording/stimulation electrode placements.

Statistics and analysis. Differences in locomotion and stereotypy due to repeated cocaine injection were analyzed using a one-way ANOVA with "injection number" (i.e., 1, 3, or 14) as a within-subject measure. Locomotion and stereotypy were also compared for saline animals for the first and fourteenth injection via $t$ test. The effects of COC or SAL treatment on the different measures of the maze task were compared using $t$ tests. For all behavioral tasks, $n=8$ per group for a total of 16 animals. For electrophysiology, one cocaine animal had misplaced electrodes and was excluded leaving $n=7$ for the cocaine group. Recorded LFP epochs were visually inspected and recordings with any artifact contamination were rejected for further analysis. Baseline differences in spontaneous and induced oscillation power and coherence were evaluated in each region using ANOVA with "treatment" (i.e., COC or SAL) as a between-subjects factor and "frequency band" as a within-subjects factor. For examining the effects of ATBS on evoked responses (i.e., N1 amplitude) in each region, treatment was a between-subjects factor, with "stimulation intensity" and "ATBS time point" (i.e., pre, post, and post $30 \mathrm{~min}$ ) as betweensubject factors. For examining the effects of ATBS on induced oscillatory activity, treatment was a between-subjects factor, with "frequency band" and ATBS time point as within-subject factors. The effects of cocaine challenge were analyzed using treatment as a between-subjects factor, and frequency band and "IV cocaine" as within-subject factors. ANOVAs were followed by Bonferroni's post hoc test corrected for multiple comparisons. Comparisons of the effects ATBS and intravenous cocaine were performed on data expressed as a percentage of baseline. If the assumption of sphericity was violated according to Mauchley's test, the Greenhouse-Geisser correction was applied and corrected $F$ values are reported. Significance was set at $p<0.05$.

\section{Results}

\section{Cocaine-induced behavioral sensitization}

Repeated cocaine administration produced robust locomotor sensitization. A common response toward the end of the dosing regimen was an immediate increase in ambulatory behavior, followed by intense stereotypy (i.e., sniffing, head-bobbing; Fig. 2). The effect of cocaine injection number on distance traveled and stereotypy counts were compared for the first, third, and last (fourteenth) injection. For distance traveled, there was a main effect of injection number $\left(F_{(2,14)}=5.73, p=0.015\right)$, such that distance traveled was significantly increased compared with the first injection after the third injection, but not the fourteenth injection. This lack of difference in distance traveled is presumably due to the rats engaging in stereotyped behaviors; there was also a main effect of injection number on stereotypy counts $\left(F_{(1.12,8.30)}=16.182, p=0.003\right)$, with a significant increase after the fourteenth injection compared with the first and third injections, which did not differ significantly. In contrast, the first and fourteenth saline injection showed no significant difference in distance traveled or stereotypy counts.

\section{Cross-maze task}

At least 4 weeks following cocaine administration, animals were tested on their ability, on consecutive days, to acquire an egocentric response using a cross maze with 1 arm blocked to form a $\mathrm{T}$ (e.g., "turn left"), retain the response on the next day, then reverse the response within the same dimension (i.e., "now turn right"), while ignoring a visual cue in the maze arm that pseudorandomly varied position between arms. On the fourth day of testing, animals were required to perform an extradimensional shift whereby they were rewarded for choosing the arm with the previously ignored visual cue. Cocaine and saline-treated animals showed no differences in the ability to learn (Fig. 3A) or retain (Fig. 3B) an egocentric response; however, COC rats showed a 
A

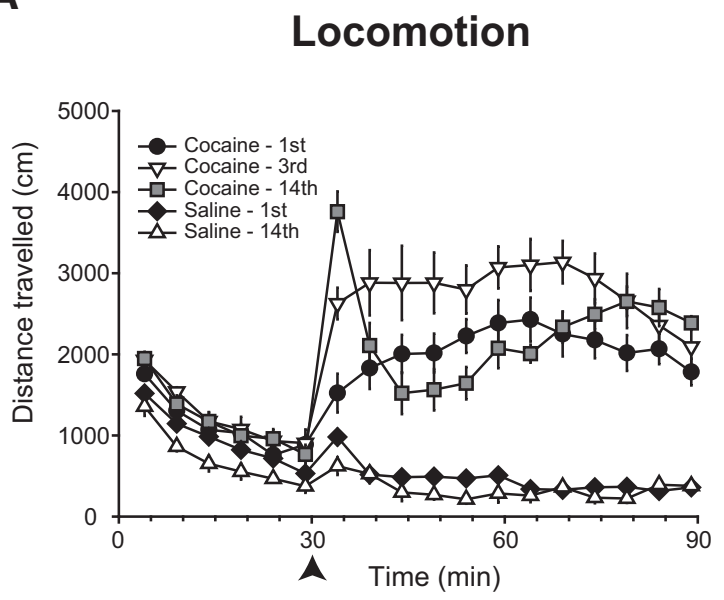

Stereotypy

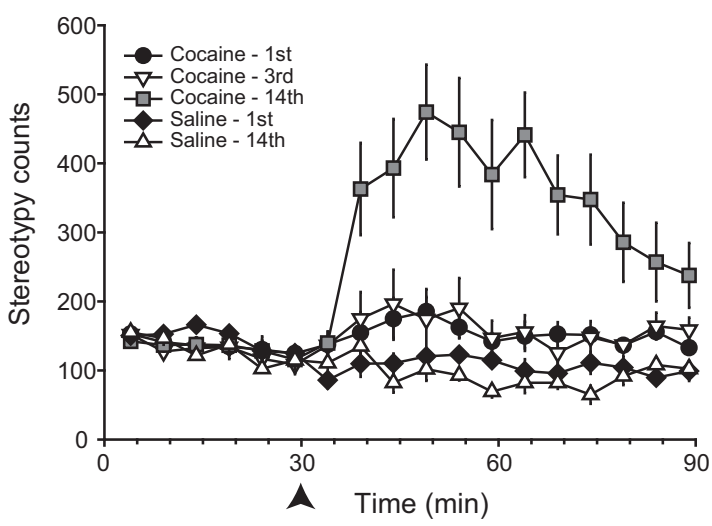

B

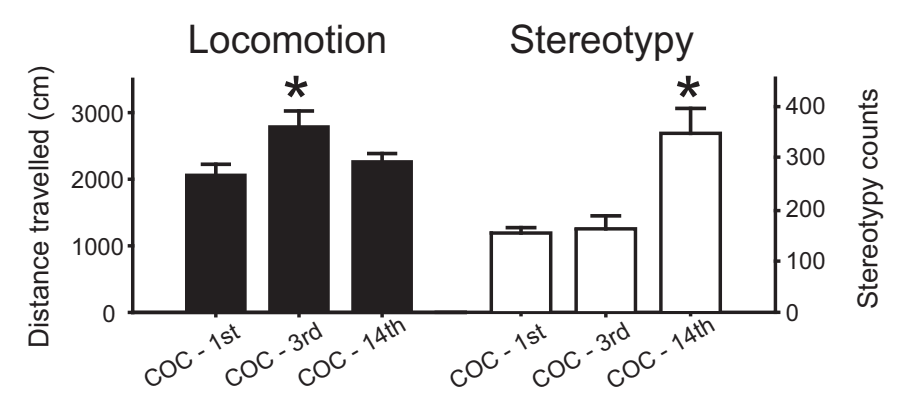

Saline

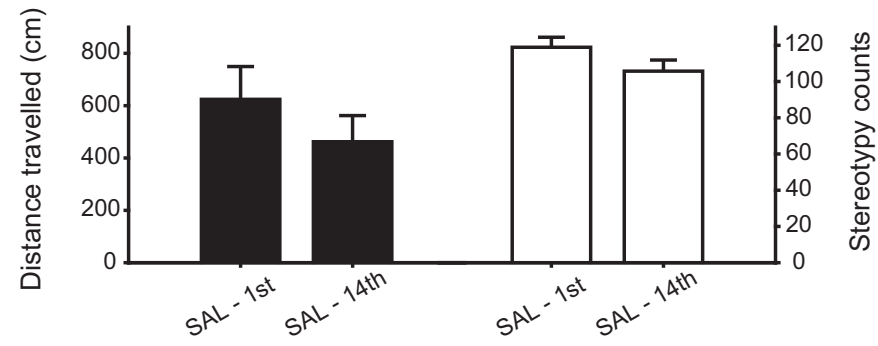

Figure 2. Repeated cocaine produces robust behavioral sensitization. $A$, Top, Locomotor response to repeated injections of $30 \mathrm{mg} / \mathrm{kg}$ cocaine, intraperitoneally, or saline. Bottom, Stereotyped movements. $\boldsymbol{B}$, Pooled group data comparing total distance traveled (left) or stereotyped movements (right) for cocaine (top) or saline (bottom)-treated groups. ${ }^{*}$, significantly different from first injection.

significantly impaired ability to reverse this association and turn in the opposite direction on the fourth day (Fig. 3C). When error types were divided into perseverative or regressive, COC animals made significantly more regressive errors while the number of preservative errors was not different between groups. No significant differences were seen between groups for any measure following an extradimensional set-shift where animals were rewarded for approaching the visual cue, which they had previously learned to ignore.

\section{LFP activity}

Between 3 and $5 \mathrm{~d}$ following behavioral testing, animals were anesthetized to assess spontaneous and evoked LFP activity. Rhythmic oscillatory fluctuations in LFP recordings are thought to reflect the summed synchronous activity of relatively large groups of neurons (Mitzdorf, 1985; Logothetis, 2003) in the vicinity of the electrode. LFP oscillations in discrete frequency bands provide an index of the predominant temporally and spatially correlated network activity in a region, and correspond to distinct behavioral states, in addition to particular aspects of neural computation (Steriade, 2006).

An obvious limitation of the current design is that the rats were anesthetized during the electrophysiological experiments; accordingly, we took a variety of different measurements de- signed to address separate dimensions of systems-level neural activity. Thus, we consider our spontaneous recordings to assess the state of the system at rest, and the evoked/induced activity to provide an index of how the system responds to activation (analogous to an organism reacting to a stimulus). In addition, examining changes in response to tetanization allows us to assess the capacity of projections to exhibit plasticity. Finally, short-latency evoked responses reflect a measure of synaptic activity after activation of an afferent pathway (i.e., the field/population EPSP), whereas examining oscillations allows us to gauge synchronous network activity within regions, in the case of power, and between regions, in the case of coherence.

\section{Spontaneous power and coherence}

Analysis of spontaneous LFPs recorded simultaneously from OFC, PFC, NAC, and BLA revealed a reduction in power in most regions that was broadband in nature but concentrated in the faster oscillation bands (Fig. 4). Thus, in OFC, power was significantly reduced in the beta and low gamma bands in COC animals compared with SAL animals [main effect of band $\left(F_{(1.00,12.01)}=10.79, p=0.007\right)$, but not treatment and no band $\times$ treatment interaction $(F<0.22, N S)]$. In PFC and NAC, low and high gamma power was significantly reduced in $\mathrm{COC}$ animals compared with SAL [PFC: main effect of band 
A

Day 1 - Response discrimination

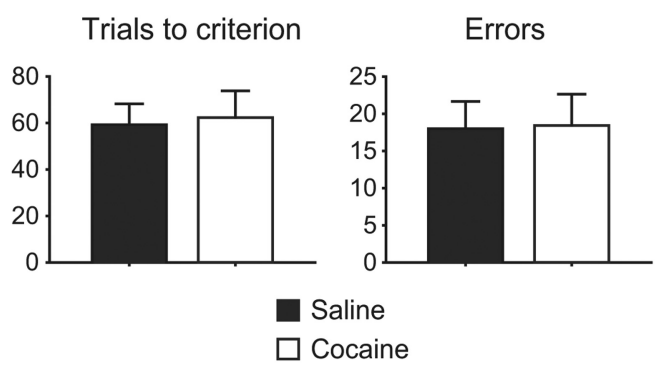

B

\section{Day 2 - Retention}

Trials to criterion

Errors

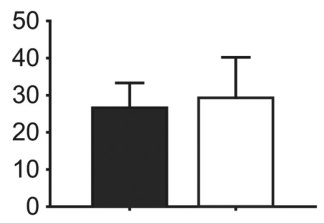

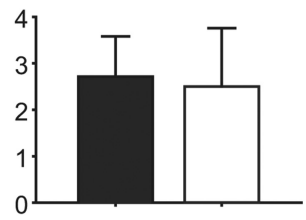

C

Day 3 - Reversal
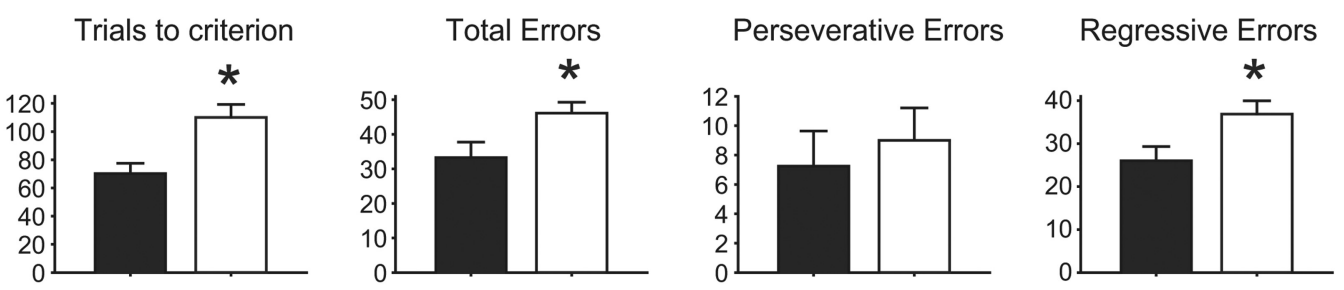

D

Day 4 - Set shift
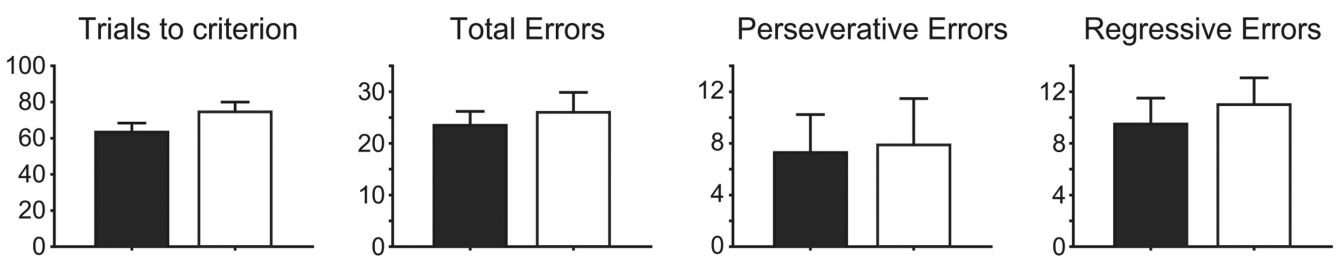

Figure 3. COC-treated animals show deficits specific to reversal learning. $\boldsymbol{A}$, No effects of treatment on initial response discrimination or $(\boldsymbol{B})$ response retention. $\boldsymbol{C}$, $C O C$ animals are significantly impaired on reversal learning measured as both total errors made or total trials to criterion. Analysis of error types revealed no difference in perseverative errors but increased regressive errors in $C O C$ animals. ${ }^{*}$, significantly different from saline-treated. $\boldsymbol{D}$, No group difference in any measure of set-shifting performance.

$\left(F_{(1.00,12.01)}=35.06, p=0.001\right)$ but not treatment and no band $\times$ treatment interaction $(F<1.14$, NS); NAC: main effect of band $\left(F_{(1.00,12.03)}=11.84, p=0.005\right.$, but not treatment and no band $\times$ treatment interaction $(F<0.40, \mathrm{NS})]$. In BLA, high gamma power was significantly lower in COC animals than SAL animals [main effect of band $\left(F_{(1.03,12.30)}=16.69, p=0.001\right)$, but not treatment and no band $\times$ treatment interaction $(F<0.03, \mathrm{NS})]$. With respect to spontaneous coherence, with all pairwise comparisons ANOVA revealed a main effect of band $(F>21.05, p<$ 0.001 , in all cases), but no significant difference between groups.

\section{Evoked/induced responses}

Acute BLA stimulation resulted in short-latency acute LFP responses in all areas. The mean latency of these responses was $9.07 \pm 0.29 \mathrm{~ms}$ in OFC, $14.34 \pm 0.52 \mathrm{in} \mathrm{PFC}$, and $12.83 \pm 0.36$ in NAC.

BLA stimulation also resulted in induced oscillations (i.e., not time locked to the stimulus) in all afferent regions, which we compared in SAL and COC animals. In OFC, COC animals had significantly higher low gamma activity than SAL animals [main effect of band $\left(F_{(1.31,15.34)}=6.48, p=0.016\right)$ but not treatment and no band $\times$ treatment interaction $(F<3.09$, NS]. In PFC, induced oscillations were significantly elevated in COC compared with SAL animals in the beta and low gamma bands [main effect of band $\left(F_{(1.53,19.86)}=6.48, p=0.038\right)$ and treatment
$\left(F_{(1,13)}=6.48, p=0.045\right)$ and a significant band by treatment interaction $\left.\left(F_{(1.53,19.86)}=6.48, p=0.047\right)\right]$. Similarly, in NAC, there was significantly greater induced oscillation power in COC animals in the beta and low and high gamma bands [main effect of band $\left(F_{(1.86,24.19)}=9.36, p=0.001\right)$ and treatment $\left(F_{(1,13)}=\right.$ $6.64, p=0.023)$ and a significant band by treatment interaction $\left.\left(F_{(1.86,24.19)}=5.14, p=0.015\right)\right]$.

BLA stimulation did not have a consistent synchronizing or desynchronizing effect on coherent activity in afferent regions, and there were no significant main effects, interactions, or SAL-COC group difference for any frequency bands (data not shown).

Thus, compared with SAL animals, COC animals had widespread reductions in spontaneous oscillation power, particularly in the faster frequency bands, and enhanced induced oscillation power in all regions. There were no differences between the groups in spontaneous or induced coherent activity (Fig. 5).

\section{Effects of ATBS}

We then compared the effect of BLA TBS on input-output curves of BLA-evoked responses between the two groups. BLA TBS significantly potentiated evoked responses at all stimulus intensities in all regions; however, there were no differences in this potentiation between COC and SAL animals (data not shown), either 
A
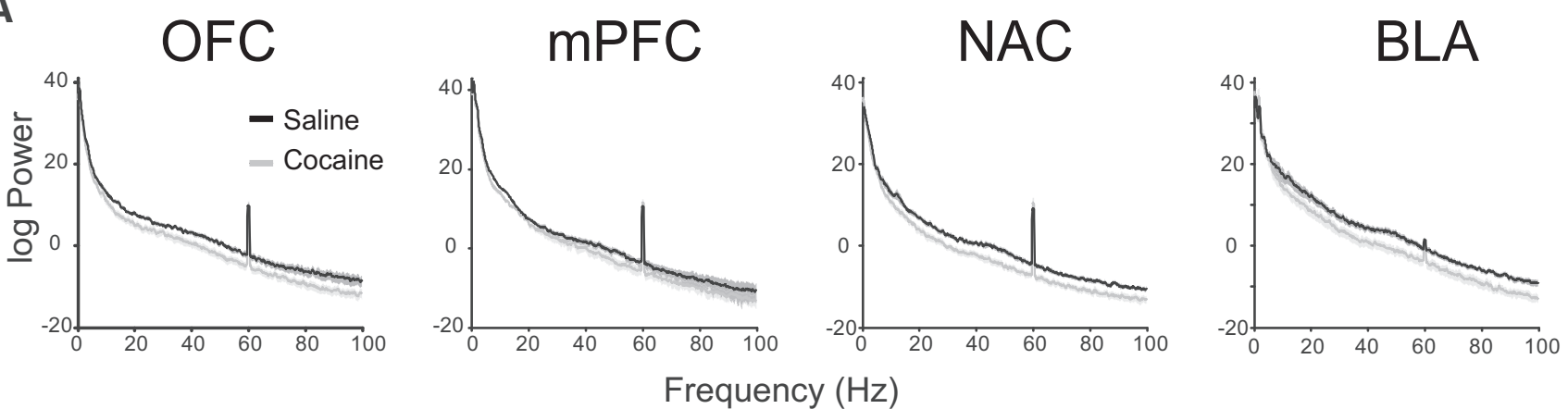

B

Saline

a Cocaine

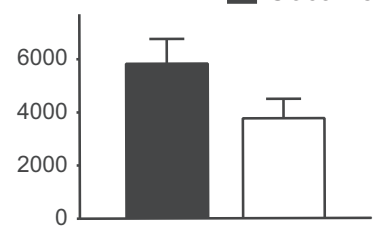

Theta $(4-12 \mathrm{~Hz})$
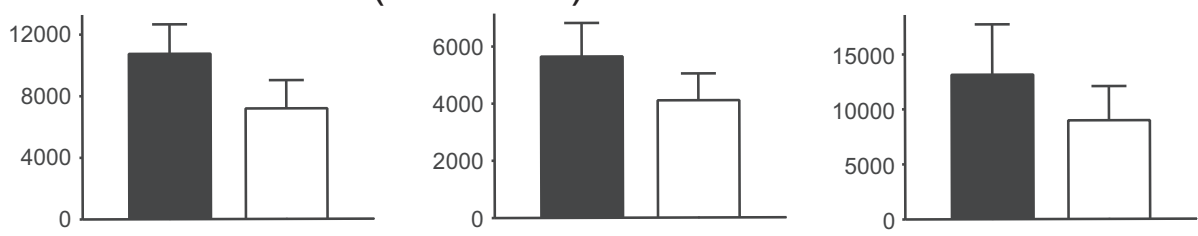

Beta $(12-30 \mathrm{~Hz})$
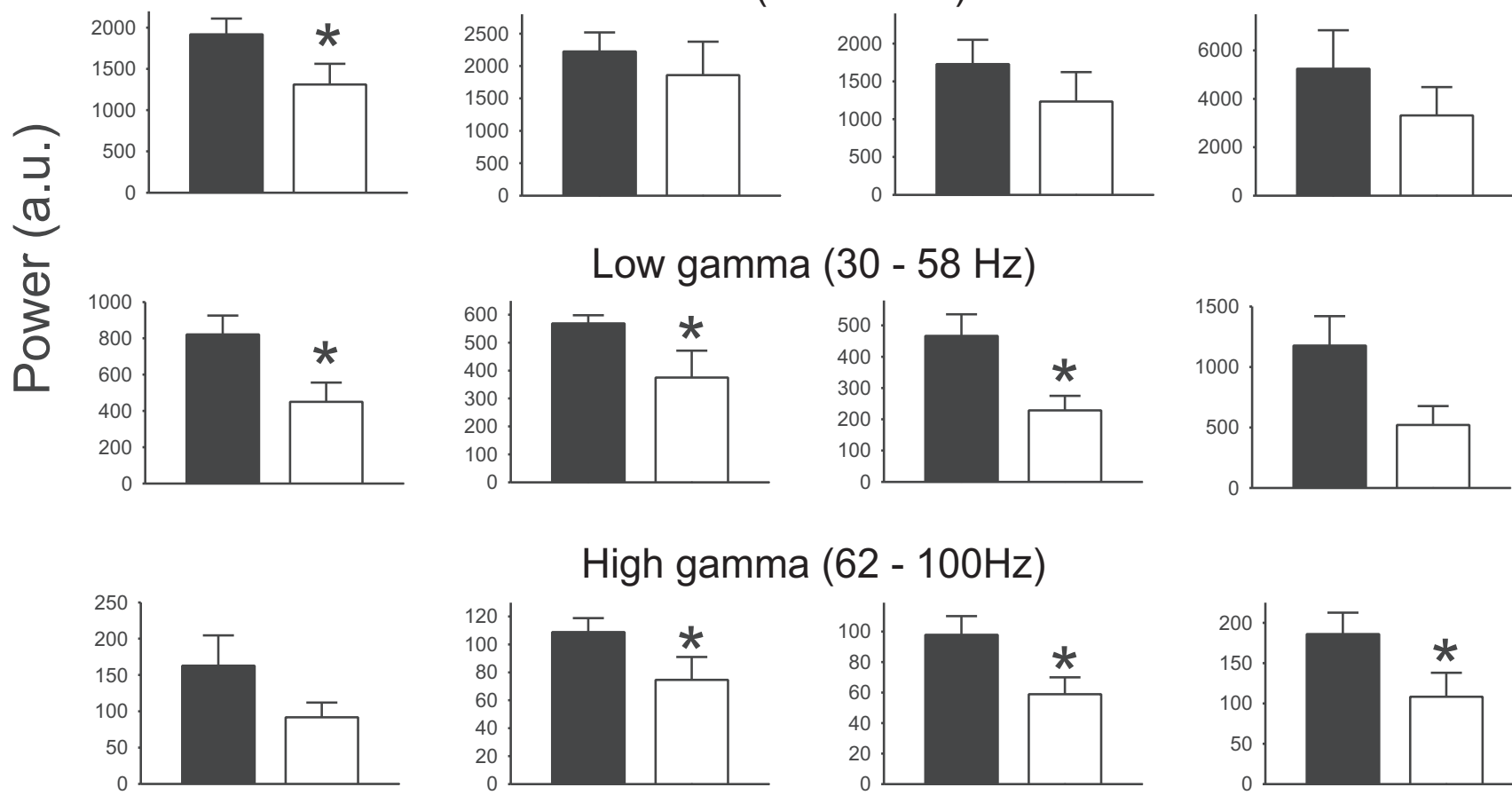

High gamma $(62-100 \mathrm{~Hz})$
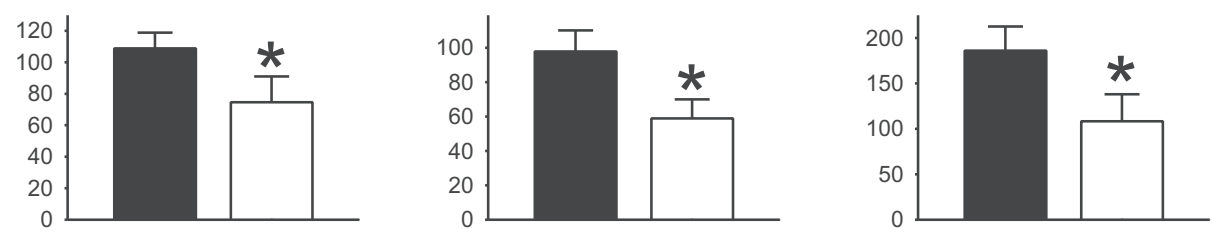

Figure 4. $C O C$ animals show reduced baseline spontaneous oscillation power, $\boldsymbol{A}$, Representative power spectra from each recorded area. $\boldsymbol{B}$, Differences in oscillation power between groups according to frequency band. ${ }^{*}$, significantly different from SAL. a.u., arbitrary units.

immediately following ATBS or 30 min post-ATBS. In addition, ATBS produced no changes in spontaneous or induced oscillatory activity at either time point (data not shown).

\section{Intravenous cocaine challenge}

Following examination of the effects of ATBS, the effects of an acute intravenous cocaine challenge on spontaneous and evoked LFP activity were assessed, using data from the ATBS post-30 time point as baseline data for the effects of cocaine challenge; data were normalized to prechallenge baseline.
Evoked responses

Intravenous cocaine produced a significant reduction in BLAevoked amplitude in all regions at all stimulation intensities (data not shown).

\section{Spontaneous power}

In general, cocaine challenge produced widespread broadband reductions in oscillation power. While cocaine challenged significantly lowered oscillation power in a number of frequency bands compared with prechallenge, this effect was not signif- 
A

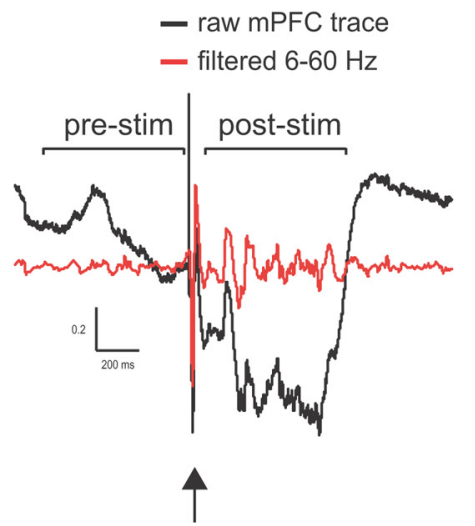

BLA stim

B

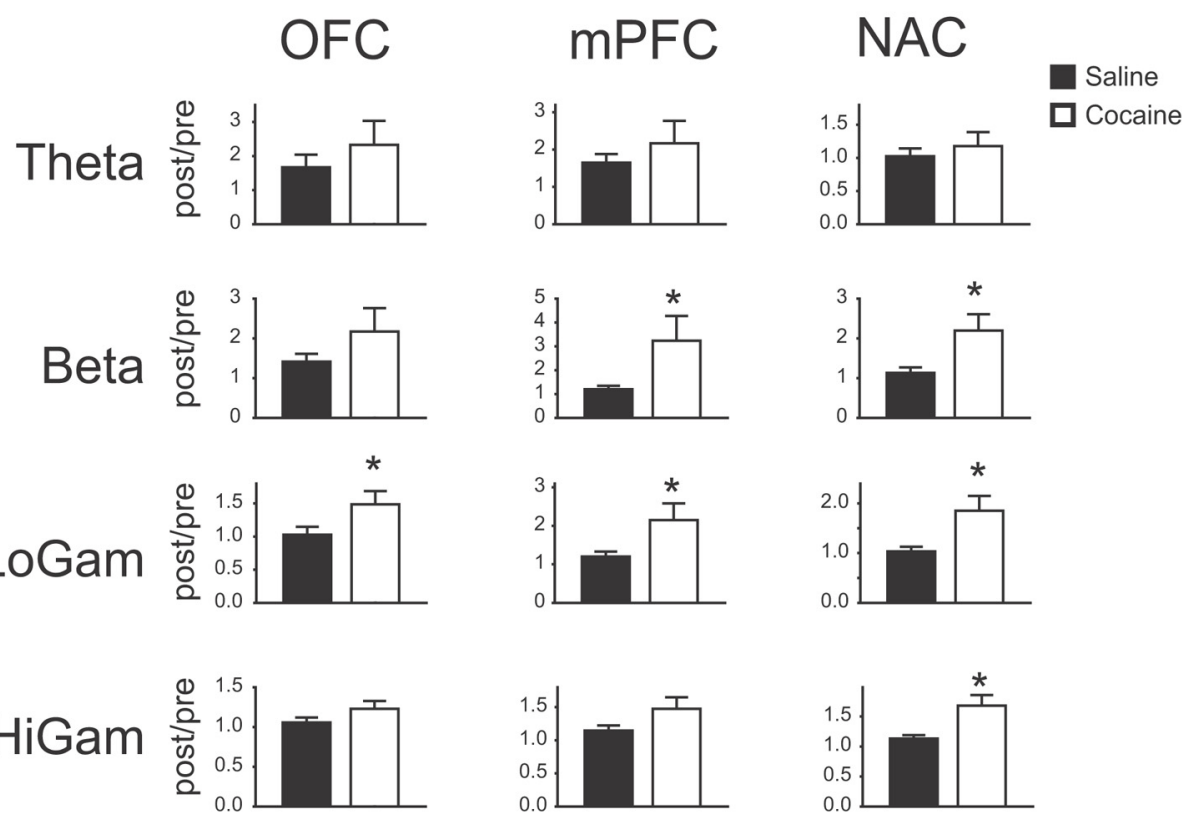

Figure 5. BLA-induced oscillations are enhanced in COC animals $A$, Left, Raw and filtered voltage traces from mPFC showing effects of acute BLA stimulation (arrow). Calibration: $200 \mathrm{~ms}, 0.2 \mathrm{mV}$. Induced oscillations were quantified by dividing power in the post-stim epoch by power in the pre-stim epoch, and then averaged over stimulus sweeps. Right, Time-frequency spectrograms showing effects of acute BLA stimulation (arrow) on raw voltage (top), traces with stimulus artifact and acute response removed (middle), and oscillation power normalized to mean prestimulus power (bottom). $\boldsymbol{B}$, Induced oscillation power in each region according to frequency band. ${ }^{*}$, significantly different from SAL.

icantly different between COC and SAL animals for any region or frequency band [OFC: main effect of cocaine challenge $\left(F_{(1,12)}=18.74, p=0.001\right)$, but not treatment or frequency band, and no interactions $(F<1.86$, NS). PFC: main effect of cocaine challenge $\left(F_{(1,12)}=5.67, p=0.033\right)$, but not treatment or frequency band, and no interactions $(F<1.35$, NS). NAC: main effect of cocaine challenge $\left(F_{(1,12)}=7.57, p=\right.$ 0.017 ), but not treatment or frequency band, and no interac- tions $(F<0.87, \mathrm{NS})$. BLA: main effect of cocaine challenge $\left(F_{(1,12)}=11.44, p=0.005\right)$, but not treatment or frequency band, and no interactions $(F<1.48, \mathrm{NS})]$. The effects of cocaine challenge on oscillatory activity according to group, region, and frequency band were similar (i.e., decreases in power) but not identical and are summarized in Figure 6. Of note, in BLA a cocaine challenge-induced reduction in power was present only in COC animals. 
A

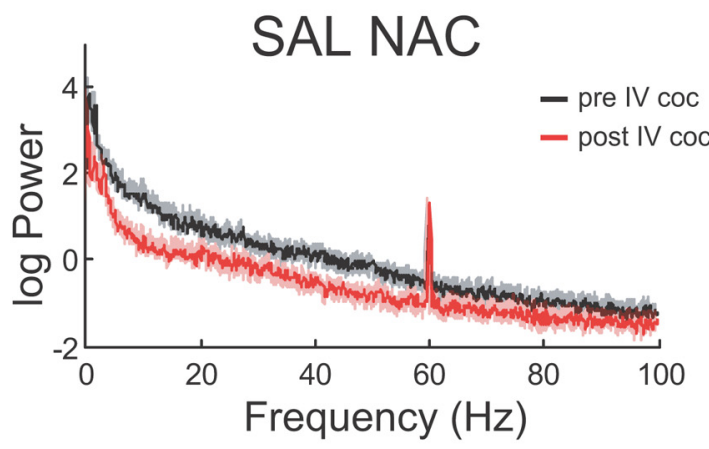

COC NAC

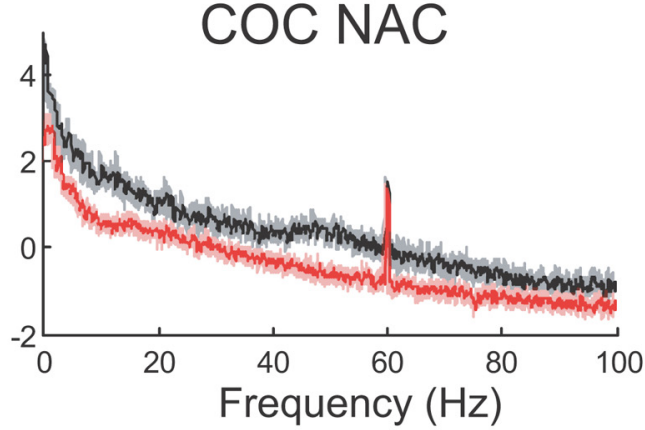

B

\begin{tabular}{|c|c|c|c|c|c|c|c|c|}
\hline & \multicolumn{2}{|c|}{ OFC } & \multicolumn{2}{|c|}{ mPFC } & \multicolumn{2}{|c|}{ NAC } & \multicolumn{2}{|c|}{ BLA } \\
\hline & SAL & $\mathrm{COC}$ & SAL & $\mathrm{COC}$ & SAL & $\mathrm{COC}$ & SAL & $\mathrm{COC}$ \\
\hline Delta & - & - & - & - & - & - & - & - \\
\hline Theta & $\downarrow$ & $\downarrow$ & - & $\downarrow$ & $\downarrow$ & $\downarrow$ & - & $\downarrow$ \\
\hline Beta & - & $\downarrow$ & - & $\downarrow$ & - & - & - & $\downarrow$ \\
\hline Lo gamma & - & - & $\downarrow$ & $\downarrow$ & $\downarrow$ & - & - & $\downarrow$ \\
\hline Hi gamma & $\downarrow$ & $\downarrow$ & - & - & $\downarrow$ & - & - & - \\
\hline
\end{tabular}

Figure 6. Cocaine challenge reduces spontaneous oscillation power in both SAL and COC groups. $A$, Representative NAC power spectrums for a SAL (left) and COC (right) animal before and after an acute $3 \mathrm{mg} / \mathrm{kg}$ intravenous cocaine challenge. Shaded area represents $95 \%$ confidence interval generated using jackknife statistics. $\boldsymbol{B}$, Within-group effects of acute cocaine challenge on spontaneous LFP power according to region and frequency band. Arrows indicate a significant change from prechallenge baseline. Horizontal line represents no change.
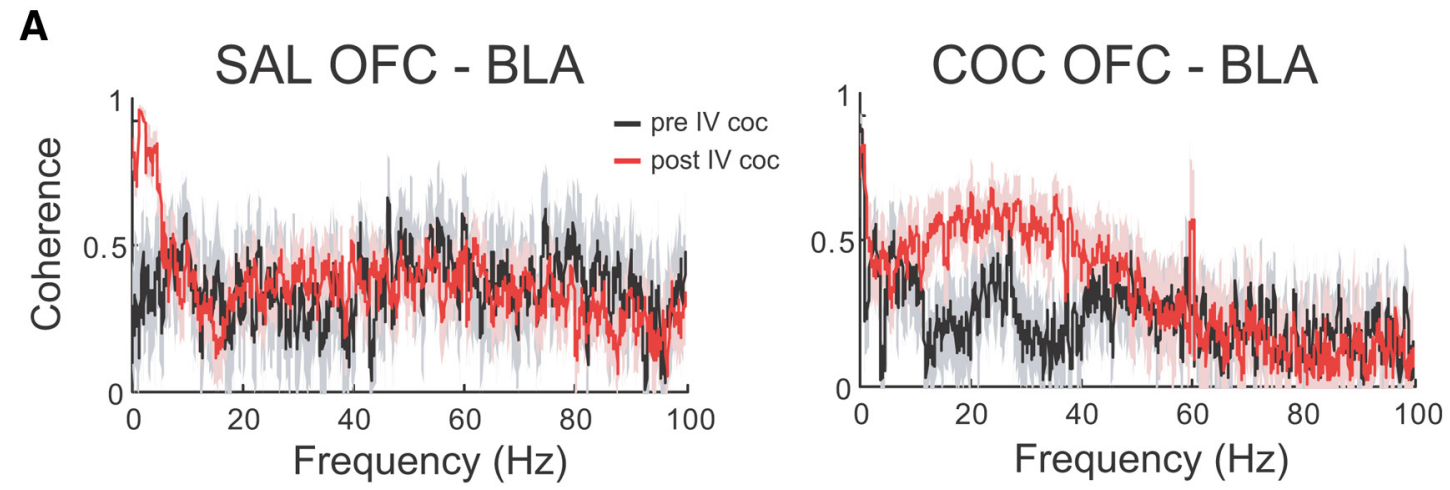

B

\begin{tabular}{|c|c|c|c|c|c|c|c|c|c|c|c|c|}
\hline \multirow[b]{3}{*}{ Delta } & \multicolumn{2}{|c|}{ OFC - BLA } & \multicolumn{2}{|c|}{ NAC - BLA } & \multicolumn{2}{|c|}{ OFC - NAC } & \multicolumn{2}{|c|}{ PFC - NAC } & \multicolumn{2}{|c|}{ OFC - NAC } & \multicolumn{2}{|c|}{ PFC - BLA } \\
\hline & SAL & $\mathrm{COC}$ & SAL & $\mathrm{COC}$ & SAL & $\mathrm{COC}$ & SAL & $\mathrm{COC}$ & SAL & $\mathrm{COC}$ & SAL & $\mathrm{COC}$ \\
\hline & $\uparrow$ & - & $\uparrow$ & - & - & - & - & - & - & - & - & - \\
\hline Theta & $\uparrow$ & $\uparrow$ & - & $\uparrow$ & - & - & - & - & - & - & - & - \\
\hline Beta & - & $\uparrow$ & - & $\uparrow$ & - & - & - & - & - & - & - & - \\
\hline Lo gamma & - & - & - & - & - & $\downarrow$ & $\downarrow$ & - & - & - & - & - \\
\hline Hi gamma & - & - & - & - & - & - & - & - & - & - & - & - \\
\hline
\end{tabular}

Figure 7. Cocaine challenge produces differential effects on spontaneous coherence in SAL versus COC animals. $A$, Representative OFC-BLA coherence plots for a SAL (left) and COC (right) animal before and after an acute $3 \mathrm{mg} / \mathrm{kg}$ intravenous cocaine challenge. Shaded area represents $95 \%$ confidence interval generated using jackknife statistics. B, Effects of acute cocaine challenge on spontaneous LFP coherence according to region and frequency band. Bold arrows indicate a significant COC/SAL difference; arrows indicate significant difference from prechallenge baseline. Horizontal line represents no change.

Spontaneous coherence

The effects of cocaine challenge on spontaneous coherence are summarized in Figure 7. There were no significant effects of cocaine challenge on OFC-PFC or PFC-BLA coherence $(F<$ $1.85, \mathrm{NS})$. OFC-NAC and PFC-NAC coherence did not differ significantly in COC versus SAL animals after cocaine chal- lenge in any band [OFC-NAC: main effect of cocaine challenge $\left(F_{(1,12)}=13.57, p=0.003\right)$, but not treatment or frequency band, and no interactions $(F<0.81, \mathrm{NS})$. PFC-NAC: main effect of cocaine challenge $\left(F_{(1,12)}=5.23, p=0.040\right)$, but not treatment or frequency band, and no interactions $(F<1.52$, NS)]. OFC-NAC coherence was significantly reduced in the 
A

\section{$\mathrm{COC}$ \\ OFC power - BLA stim}

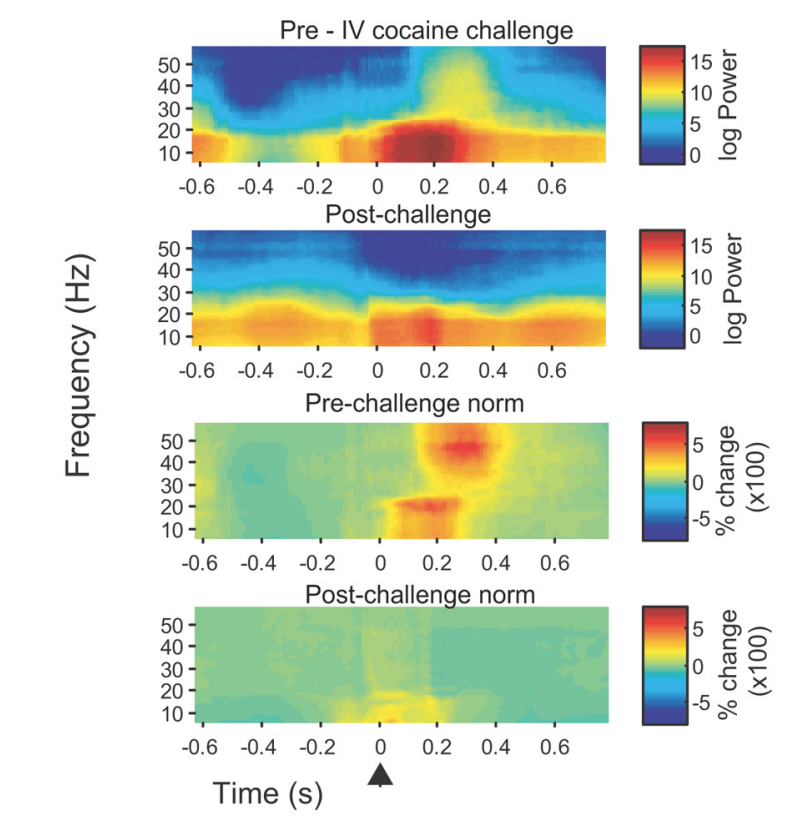

B
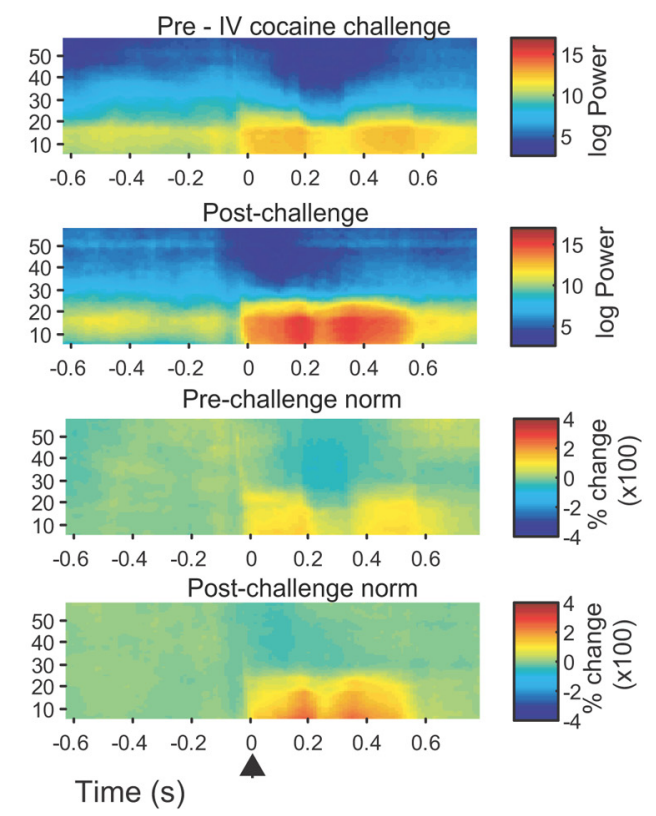

\begin{tabular}{|c|c|c|c|c|c|c|c|c|c|c|c|c|}
\hline & \multicolumn{2}{|c|}{ OFC } & \multicolumn{2}{|c|}{ PFC } & \multicolumn{2}{|c|}{ NAC } & \multicolumn{2}{|c|}{ OFC - NAC } & \multicolumn{2}{|c|}{ OFC - PFC } & \multicolumn{2}{|c|}{ PFC - NAC } \\
\hline & SAL & $\mathrm{COC}$ & SAL & $\mathrm{COC}$ & SAL & $\mathrm{COC}$ & SAL & $\mathrm{COC}$ & SAL & $\mathrm{COC}$ & SAL & $\mathrm{COC}$ \\
\hline Theta & $\uparrow$ & - & $\uparrow$ & - & $\uparrow$ & - & $\downarrow$ & - & - & - & - & - \\
\hline Beta & - & $\downarrow$ & - & $\downarrow$ & - & $\downarrow$ & - & $\downarrow$ & - & - & - & - \\
\hline Lo gamma & - & $\downarrow$ & - & $\downarrow$ & - & $\downarrow$ & - & - & - & - & - & - \\
\hline Hi gamma & - & $\downarrow$ & - & $\downarrow$ & - & $\downarrow$ & - & - & - & - & - & - \\
\hline
\end{tabular}

Figure 8. Cocaine challenge produces selective alterations in induced oscillation power and coherence in COC and SAL animals $A$, Raw (top) and normalized (bottom) representative timefrequency spectrograms showing effects of cocaine challenge on BLA-induced oscillations in OFC in a COC (left) and SAL (right) animal. Arrowhead shows time of BLA stimulation. B, Effects of acute cocaine challenge on induced LFP power and coherence according to region and frequency band. Bold arrows indicate a significant COC/SAL difference; arrows indicate significant difference from prechallenge baseline. Horizontal line represents no change.

low gamma band compared with prechallenge in COC but not SAL animals, and PFC-NAC coherence was significantly reduced in the low gamma band in SAL animals only.

There were a number of changes in spontaneous OFC-BLA coherence following cocaine challenge. OFC-BLA coherence was significantly increased in the delta band in SAL animals compared with COC animals and to SAL prechallenge, and significantly increased in the beta band in COC animals compared with SAL animals and to COC prechallenge [main effect of cocaine challenge $\left(F_{(1,12)}=14.57, p=0.002\right)$ and significant cocaine challenge $\times$ frequency band interaction $\left(F_{(2.24,26.85)}=3.97, p=\right.$ 0.027 ), no effect of treatment or frequency band $(F<3.00$, NS)]. In addition to the within-subject effects mentioned above, OFCBLA coherence was also significantly elevated compared with prechallenge in the theta band in both COC and SAL animals.

NAC-BLA coherence was affected in a qualitatively similar way to OFC-BLA coherence. Postchallenge, NAC-BLA coherence was significantly elevated in the delta band in SAL animals compared with both COC animals and SAL prechallenge [main effect of cocaine challenge $\left(F_{(1,12)}=15.65, p=0.002\right)$, but not treatment or frequency band, and no interactions $(F<2.31$, NS)]. Furthermore, in COC animals, NAC-BLA coherence was significantly elevated in the theta and beta compared with prechallenge.
Induced oscillations

Cocaine challenge produced widespread changes in induced oscillatory activity that were, for the most part, similar between regions (Fig. 8). In all regions, there were significant reductions in induced beta and low and high gamma power in COC animals compared with both SAL animals and to COC animals prechallenge. In contrast, induced theta power was significantly elevated in SAL animals in OFC and PFC compared with prechallenge, and in NAC compared with both prechallenge and COC animals. [OFC: main effect of frequency band $\left(F_{(1.45,18.90)}=9.55, p=0.002\right)$ and significant cocaine challenge $\times$ frequency band interaction $\left(F_{(1.10,14.28)}=10.37, p=0.005\right)$; no effects of treatment, cocaine challenge, and no other interactions $(F<$ $4.58, \mathrm{NS})$. PFC: main effect of frequency band $\left(F_{(1.13,14.73)}=12.33\right.$, $p=0.003)$ and significant cocaine challenge $\times$ frequency band interaction $\left(F_{(1.14,14.80)}=13.43, p=0.002\right)$; no effects of treatment, cocaine challenge, and no other interactions $(F<4.37$, NS). NAC: main effect of frequency band $\left(F_{(2.28,29.58)}=5.98, p=0.005\right)$; significant cocaine challenge $\times$ frequency band $\left(F_{(1.88,24.47)}=6.76, p=\right.$ $0.005)$ and treatment $\times$ cocaine challenge interactions $\left(F_{(1,12)}=\right.$ $10.97, p=0.006)$; no effect of treatment or cocaine challenge, and no other significant interactions $(F<3.50, \mathrm{NS})]$.

Induced OFC-NAC coherence was significantly reduced in COC animals compared with both SAL animals and prechallenge 
Table 1. Summary of differences in spontaneous and induced oscillation power and coherence at baseline and following intravenous cocaine challenge

\begin{tabular}{lll}
\hline Baseline & COC animals have $\downarrow$ spontaneous fast oscillations and $\uparrow$ induced fast oscillations compared to SAL (all regions) \\
\hline Intravenous cocaine challenge & Spontaneous power & Broadband $\downarrow$ in most regions in both COC and SAL (except BLA, $\downarrow$ only in COC) \\
& Induced power & $\uparrow$ induced theta power in SAL (all regions) \\
& $\downarrow$ induced fast oscillation power in COC (all regions) \\
& $\uparrow$ delta/theta coherence in SAL (OFC-BLA, BLA-NAC only) \\
& $\uparrow$ theta/beta coherence in COC (OFC-BLA, BLA-NAC only) \\
& $\downarrow$ induced theta coherence in SAL (OFC-NAC) \\
& $\downarrow$ induced coherence & \\
& & induced beta coherence in COC (OFC-NAC) \\
\hline
\end{tabular}

in the beta and low gamma bands [main effect of cocaine challenge $\left(F_{(1,12)}=30.30, p<0.001\right)$ no other main effects or interactions $(F<0.97, \mathrm{NS})]$. With respect to within-subject effects, in COC animals induced high gamma coherence was significantly reduced compared with prechallenge, and in SAL animals induced theta coherence was significantly reduced compared with prechallenge. There were no significant main effects, interactions, or group differences in induced coherence between PFCNAC and OFC-PFC $(F<1.55$, NS).

\section{Discussion}

Cocaine abuse is associated with long-lasting deficits in executive function and behavioral flexibility. Here we report that a sensitizing regimen of cocaine administration that produces persistent deficits in reversal learning but not set-shifting is associated with abnormal spontaneous and induced LFP oscillatory activity along a limbic cortico-striatal circuit known to be affected by cocaine (summarized in Table 1).

\section{Behavior}

The dosing regimen used here produced pronounced locomotor sensitization in COC animals; on a maze-based task these animals exhibited a selective impairment in reversal learning. A breakdown of the error types made by COC animals during reversal suggests that the deficits may relate more to an inability to maintain the use of correct strategy rather than pure perseveration (Ragozzino, 2002; Floresco et al., 2006b). Notably, a similar error profile was observed in response to OFC inactivation in a version of the task used here (Ghods-Sharifi et al., 2008).

These data are consistent with previous reports that an identical regimen of noncontingent cocaine produces deficits in an odor-based go-no go reversal task (Schoenbaum et al., 2004). Notably, the reversal impairments in that study are almost identical in magnitude to those following self-administered cocaine (Calu et al., 2007). Similarly, monkeys with extensive cocaine self-administration history are also impaired on a reversal learning task (Porter et al., 2011). Interestingly, we saw no deficits in an extradimensional set-shift, in contrast to rats treated with a different dosing regimen (Goto and Grace, 2005). Of note, no animal studies to date have examined set-shifting following self-administered cocaine; the possibility exists that self-administered cocaine may produce deficits in set-shifting that are not apparent following noncontingent cocaine.

The anatomical and neurochemical substrates underlying reversal learning have been relatively well studied. The lateral OFC plays a critical role in reversal learning in mice, rats, monkeys, and humans. Notably, OFC-lesioned rats show reversal impairments that are almost indistinguishable from cocaine-treated rats (for review, see Schoenbaum et al., 2009). More important than OFC alone, however, appears to be OFC-BLA interactions. Although BLA lesions do not impair reversal learning, they can rescue the performance of OFC-lesioned rats (Stalnaker et al., 2007a). In addition, aberrant coding in BLA neurons is thought to be the proximal cause of reversal-related impairments in OFC in cocaine-experienced rats (Stalnaker et al., 2007b). In contrast, the rat PFC is heavily implicated in set-shifting (Ragozzino et al., 1999; Floresco et al., 2006b), but is thought to be minimally involved in reversal learning-and vice versa (McAlonan and Brown, 2003; Ghods-Sharifi et al., 2008). Data regarding the role of the NAC are less consistent, with reports of both deficits (Stern and Passingham, 1995; Ferry et al., 2000) and no effect (Schoenbaum and Setlow, 2003; Castañé et al., 2010) on reversal following lesions in animals. Human imaging studies have shown activation in NAC during reversal errors (Cools et al., 2002) and during punishment-based reversal (Robinson et al., 2010).

Our behavioral results suggested that COC animals had deficits in OFC-BLA-related processing. We hypothesized that ATBS-induced LTP-like plasticity of short-latency, presumably monosynaptic BLA $\rightarrow$ OFC evoked responses would be impaired/ occluded in COC animals, analogous to the observation that stress blocks ATBS-induced potentiation of BLA-evoked responses in PFC (Maroun and Richter-Levin, 2003). BLA stimulation produced robust evoked responses in all three regions that were significantly potentiated following ATBS; however, there were no differences in this potentiation in COC and SAL animals in any region, suggesting that the proposed BLA-OFC LTP impairment in COC animals did not take place.

We observed a number of group differences in spontaneous LFP oscillations and those induced by acute BLA stimulation. In COC animals, there was a widespread and broadband suppression of oscillatory activity. When analyzed according to frequency band, all regions showed significant reductions in the higher frequency bands. These data correspond well with studies showing that abstinent cocaine users have reduced cortical electroencephalographic (EEG) power (Noldy et al., 1994) and lowered frontostriatal glucose utilization (Volkow et al., 1992). With respect to induced activity; COC animals showed increased induced oscillation power in the beta and gamma bands compared with the immediate prestimulus epoch. It is unclear whether this reflects an enhanced stimulus-induced synchronization, or the lower oscillation power at baseline in COC animal. There were no group differences with respect to spontaneous or induced coherent activity between any region pairs. If basic synchronization mechanisms were impaired in COC, one would expect reduction in both spontaneous and evoked oscillations, suggesting that the observed changes instead reflect changes in gain or dynamic range of the network at rest versus when activated. Together, the observed changes suggest substantial changes in the mechanisms underlying synchronization in the faster frequency bands.

\section{Cocaine challenge}

Cocaine challenge reduced the amplitude of BLA-evoked responses in all regions. Similar to what was observed with ATBS, the magnitude of these changes did not differ in COC and SAL animals. 
Spontaneous power and coherence

Cocaine challenge also suppressed broadband spontaneous oscillatory power in all regions, extending previous findings showing that acute cocaine reduces cortical EEG synchronization in both awake and anesthetized rats (Ferger et al., 1996; Chang et al., 1998). Both COC and SAL animals showed significant reductions in power compared with prechallenge baseline in all regions; while the pattern of changes was similar, the bands with statistically significant changes varied according to region. These data are consistent with a report by Ferger et al. (1996) showing that acute cocaine administration desynchronizes cortical EEG activity, leading to reduced baseline EEG power in sensitized rats, which is further reduced upon cocaine challenge. Notably, in COC animals BLA power was significantly reduced in the theta, beta, and low gamma bands following challenge; there were no significant effects in SAL animal BLA power. These effects may relate to the observed aberrant BLA encoding that is the suggested to be the proximal cause of cocaine-dependent impaired reversal learning (Stalnaker et al., 2007b).

The most prominent and specific effects of cocaine challenge were on OFC-BLA coherence; cocaine challenge significantly increased coherence in the delta and theta bands in SAL animals. In contrast, in COC animals a different pattern of changes emerged, with significant increases in theta and beta coherence but no changes in delta coherence. Similar effects were observed with NAC-BLA coherence, and COC animals also showed a reduction in OFC-NAC gamma coherence. In contrast, there were no effects on coherence between any other regions, further arguing for specific and selective functional adaptations in COC animals.

\section{Induced power and coherence}

Cocaine challenge produced changes in induced oscillation power that were similar in all regions for each group, but differed between groups. Thus, cocaine challenge significantly enhanced induced theta oscillations in SAL animals but not COC animals, and significantly reduced induced oscillations in the faster frequency bands in COC but not SAL animals. These findings are notable due to the frequency-band specificity and the opposite direction of changes. Our data suggest that cocaine facilitates theta generation in response to stimulus; as theta oscillations are often used as an index of arousal (Buzsaki, 2002), this idea is consistent with the idea of cocaine increasing arousal. The reductions in induced fast oscillations in COC animals may represent a compensatory mechanism in response to the enhanced induced theta oscillations produced by cocaine in SAL animals. An alternative explanation is that cocaine is normalizing the drug-withdrawn system-abstinent COC animals have elevated induced fast oscillations compared with SAL animals, which an acute cocaine dose returns to control levels. This idea is consistent with the normalization-type effect of cocaine on frontal blood oxygenation level-dependent metabolism (Garavan et al., 2008) and cognitive performance (Woicik et al., 2009) in abstinent chronic users. What might underlie the transition in effects of cocaine on naive versus abstinent, sensitized animals? One possibility is that, after repeated dosing, the reduction in broadband power becomes permanent, leading to the lower spontaneous power seen in COC animals at baseline, similar to the effects of repeated cocaine on EEG activity (Ferger et al., 1996). To compensate for the reduced synchronization, the system becomes more sensitive to activation, leading to the elevated fast oscillations in COC animal.

In general, we observed a dissociation of evoked responses and oscillations, with COC/SAL group differences in oscillations not reflected in evoked responses. Both short-latency evoked LFP responses and LFP oscillations depend on synchrony-synchronous activation of an afferent pathway in the case of the former and synchronous rhythmic membrane potential fluctuation in the case of the latter. Oscillatory activity is often generated by recurrent activity within and between circuits (Steriade, 2006), and it is the underlying mechanisms of oscillation generation that appear to be affected by cocaine to a greater extent than synaptic function (although the two may not be entirely dissociablechanges in synaptic function could conceivably affect network activity and oscillations). Faster oscillations are thought to rely on synchronized interneuron activity, and aberrant plasticity in interneuron-coordinated network activity in cocaine animals may play a significant role in the observed changes.

A potential limitation of the present study is the fact that electrophysiological measurements were performed under anesthesia, raising the issue as to whether these data translate to the awake state. However, dopamine-depleted rats show prominent spontaneous cortical and subcortical beta oscillations in both awake and anesthetized states (Mallet et al., 2008a,b), and furthermore, Ahrens and Freeman (2001) reported similar profiles of electrically evoked entorhinal cortex LFP activity in awake and anesthetized rats, supporting the notion that that our data are relevant for awake animals.

\section{Summary}

Taken as a whole, we can broadly classify the observed changes in LFP activity in three ways. First, there are changes in activity stemming from various manipulations that do not differ between COC and SAL groups: ATBS potentiation of BLA-evoked responses, acute cocaine-induced suppression of BLA-evoked responses, and cocaine-induced suppression of spontaneous oscillations. Then there are differences in activity in COC and SAL animals that are generally similar in all recorded regions: altered baseline spontaneous and induced fast oscillations and effects of cocaine challenge on spontaneous and evoked power. Finally, there are the differences between COC and SAL groups that are regionally specific: effects of cocaine challenge on spontaneous and induced coherence. With respect to the functional significance of LFP data, this last category is of particular interest, as the changes were restricted to OFC-BLA, BLA-NAC, and to a much lesser extent, OFC-NAC-all regions previously implicated in cocaine-induced reversal deficits similar to those we observed. Acute cocaine enhanced OFC-BLA coherence in the slower frequency bands (i.e., delta/theta) in SAL animals and the faster frequency bands in COC animals. What, then, is the significance of the shift in coherent OFC-BLA activity from slower to faster frequencies? Functionally, this transition may signify a shift in how neuronal ensemble coding is affected by cocaine. In OFC, separate populations of neurons phase lock to theta and gamma oscillations (van Wingerden et al., 2010), and a modeling study suggests that excitatory neuronal populations drive lower frequency oscillations, whereas cortical fast-spiking interneurons can enhance fast oscillatory activity by selectively suppressing lower frequency activity (Neymotin et al., 2011). The shift in frequency of OFC-BLA coherence may therefore represent a selective change in the neuronal populations affected by cocaine. This in turn may reflect a pathological progression occurring with repeated cocaine exposure, with increases in synchrony shifting from slower oscillations related to general arousal and activity state (Buzsaki, 2002; Steriade, 2006) to faster oscillations related to cognition (Tallon-Baudry, 2003; Schnitzler and Gross, 2005). This is consistent with studies showing that the initial 
effects of cocaine on cerebral glucose utilization are restricted to limbic and reward-related regions, but following chronic use expand to affect higher order cognitive and associative areas (Porrino et al., 2007) that may represent interference with flexible interpretation of information.

Functionally, this altered synchronization may relate to the changes in associative encoding in BLA and OFC in cocaineexperienced rats during reversal learning (Stalnaker et al., 2006; Stalnaker et al., 2007a,b). One possibility is that it reflects a pathological "locking-in," which impairs the flexible updating of outcome representations as contingencies change, resulting in an inability to change strategies. Supporting this idea, a recent imaging study reported heightened functional connectivity in a frontal cortical network associated with reversal learning deficits in cocaine abusers (Camchong et al., 2011). An alternative interpretation, based on the fact that lower frequency oscillations are synchronizing over greater distances than faster oscillations (Destexhe et al., 1999), is that there is actually less OFC-BLA functional connectivity and interaction following repeated cocaine exposure.

\section{Implications}

These data indicate that repeated exposure to cocaine can produce changes in synchronized network activity along limbic cortico-striatal circuits that persist long into abstinence. Furthermore, the regional specificity of these changes strongly suggests that they underlie the impairments in reversal learning. Aberrant synchronization within and between regions, and consequent dysregulation of the neurocircuitry involved in executive control, may contribute to the long-lasting maladaptive decision making seen in cocaine abusers.

\section{References}

Ahrens KF, Freeman WJ (2001) Response dynamics of entorhinal cortex in awake, anesthetized, and bulbotomized rats. Brain Res 911:193-202. CrossRef Medline

Ardila A, Rosselli M, Strumwasser S (1991) Neuropsychological deficits in chronic cocaine abusers. Int J Neurosci 57:73-79. CrossRef Medline

Birrell JM, Brown VJ (2000) Medial frontal cortex mediates perceptual attentional set shifting in the rat. J Neurosci 20:4320-4324. Medline

Bolla KI, Rothman R, Cadet JL (1999) Dose-related neurobehavioral effects of chronic cocaine use. J Neuropsychiatry Clin Neurosci 11:361-369. Medline

Bonson KR, Grant SJ, Contoreggi CS, Links JM, Metcalfe J, Weyl HL, Kurian V, Ernst M, London ED (2002) Neural systems and cue-induced cocaine craving. Neuropsychopharmacology 26:376-386. CrossRef Medline

Boulougouris V, Dalley JW, Robbins TW (2007) Effects of orbitofrontal, infralimbic and prelimbic cortical lesions on serial spatial reversal learning in the rat. Behav Brain Res 179:219-228. CrossRef Medline

Buzsaki G (2002) Theta oscillations in the hippocampus. Neuron 33:325-340. Medline

Calu DJ, Stalnaker TA, Franz TM, Singh T, Shaham Y, Schoenbaum G (2007) Withdrawal from cocaine self-administration produces long-lasting deficits in orbitofrontal-dependent reversal learning in rats. Learn Mem 14: 325-328. CrossRef Medline

Camchong J, MacDonald AW 3rd, Nelson B, Bell C, Mueller BA, Specker S, Lim KO (2011) Frontal hyperconnectivity related to discounting and reversal learning in cocaine subjects. Biol Psychiatry 69:1117-1123. CrossRef Medline

Carelli RM, Williams JG, Hollander JA (2003) Basolateral amygdala neurons encode cocaine self-administration and cocaine-associated cues. J Neurosci 23:8204-8211. Medline

Cassell MD, Wright DJ (1986) Topography of projections from the medial prefrontal cortex to the amygdala in the rat. Brain Res Bull 17:321-333. CrossRef Medline

Cassell MD, Chittick CA, Siegel MA, Wright DJ (1989) Collateralization of the amygdaloid projections of the rat prelimbic and infralimbic cortices. J Comp Neurol 279:235-248. CrossRef Medline
Castañé A, Theobald DE, Robbins TW (2010) Selective lesions of the dorsomedial striatum impair serial spatial reversal learning in rats. Behav Brain Res 210:74-83. CrossRef Medline

Chang AY, Chan JY, Tsen LY, Chan SH (1998) Differential participation of hippocampal formation in cocaine-induced cortical electroencephalographic desynchronization and penile erection in the rat. Synapse 30: 140-149. CrossRef Medline

Childress AR, Mozley PD, McElgin W, Fitzgerald J, Reivich M, O’Brien CP (1999) Limbic activation during cue-induced cocaine craving. Am J Psychiatry 156:11-18. Medline

Chudasama Y, Robbins TW (2003) Dissociable contributions of the orbitofrontal and infralimbic cortex to pavlovian autoshaping and discrimination reversal learning: further evidence for the functional heterogeneity of the rodent frontal cortex. J Neurosci 23:8771-8780. Medline

Colzato LS, Huizinga M, Hommel B (2009) Recreational cocaine polydrug use impairs cognitive flexibility but not working memory. Psychopharmacology 207:225-234. CrossRef Medline

Cools R, Clark L, Owen AM, Robbins TW (2002) Defining the neural mechanisms of probabilistic reversal learning using event-related functional magnetic resonance imaging. J Neurosci 22:4563-4567. Medline

Destexhe A, Contreras D, Steriade M (1999) Spatiotemporal analysis of local field potentials and unit discharges in cat cerebral cortex during natural wake and sleep states. J Neurosci 19:4595-4608. Medline

Ersche KD, Roiser JP, Robbins TW, Sahakian BJ (2008) Chronic cocaine but not chronic amphetamine use is associated with perseverative responding in humans. Psychopharmacology 197:421-431. CrossRef Medline

Ferger B, Stahl D, Kuschinsky K (1996) Effects of cocaine on the EEG power spectrum of rats are significantly altered after its repeated administration: do they reflect sensitization phenomena? Naunyn Schmiedebergs Arch Pharmacol 353:545-551. Medline

Ferry AT, Lu XC, Price JL (2000) Effects of excitotoxic lesions in the ventral striatopallidal-thalamocortical pathway on odor reversal learning: inability to extinguish an incorrect response. Exp Brain Res 131:320-335. CrossRef Medline

Fillmore MT, Rush CR (2006) Polydrug abusers display impaired discrimination-reversal learning in a model of behavioural control. J Psychopharmacol 20:24-32. Medline

Finch DM (1996) Neurophysiology of converging synaptic inputs from the rat prefrontal cortex, amygdala, midline thalamus, and hippocampal formation onto single neurons of the caudate/putamen and nucleus accumbens. Hippocampus 6:495-512. CrossRef Medline

Floresco SB, Ghods-Sharifi S, Vexelman C, Magyar O (2006a) Dissociable roles for the nucleus accumbens core and shell in regulating set shifting. J Neurosci 26:2449-2457. CrossRef Medline

Floresco SB, Magyar O, Ghods-Sharifi S, Vexelman C, Tse MT (2006b) Multiple dopamine receptor subtypes in the medial prefrontal cortex of the rat regulate set-shifting. Neuropsychopharmacology 31:297-309. CrossRef Medline

Garavan H, Morgan RE, Mactutus CF, Levitsky DA, Booze RM, Strupp BJ (2000) Prenatal cocaine exposure impairs selective attention: evidence from serial reversal and extradimensional shift tasks. Behav Neurosci 114: 725-738. CrossRef Medline

Garavan H, Kaufman JN, Hester R (2008) Acute effects of cocaine on the neurobiology of cognitive control. Philos Trans R Soc Lond B Biol Sci 363:3267-3276. CrossRef Medline

Ghods-Sharifi S, Haluk DM, Floresco SB (2008) Differential effects of inactivation of the orbitofrontal cortex on strategy set-shifting and reversal learning. Neurobiol Learn Mem 89:567-573. CrossRef Medline

Gill KM, Grace AA (2011) Heterogeneous processing of amygdala and hippocampal inputs in the rostral and caudal subregions of the nucleus accumbens. Int J Neuropsychopharmacol 14:1301-1314. CrossRef Medline

Gillen RW, Kranzler HR, Bauer LO, Burleson JA, Samarel D, Morrison DJ (1998) Neuropsychologic findings in cocaine-dependent outpatients. Prog Neuropsychopharmacol Biol Psychiatry 22:1061-1076. CrossRef Medline

Goldstein RZ, Leskovjan AC, Hoff AL, Hitzemann R, Bashan F, Khalsa SS, Wang GJ, Fowler JS, Volkow ND (2004) Severity of neuropsychological impairment in cocaine and alcohol addiction: association with metabolism in the prefrontal cortex. Neuropsychologia 42:1447-1458. CrossRef Medline

Goto Y, Grace AA (2005) Dopamine-dependent interactions between lim- 
bic and prefrontal cortical plasticity in the nucleus accumbens: disruption by cocaine sensitization. Neuron 47:255-266. CrossRef Medline

Groenewegen HJ, Trimble M (2007) The ventral striatum as an interface between the limbic and motor systems. CNS Spectr 12:887-892. Medline

Groenewegen HJ, Wright CI, Beijer AV, Voorn P (1999) Convergence and segregation of ventral striatal inputs and outputs. Ann N Y Acad Sci 877: 49-63. CrossRef Medline

Hanlon CA, Dufault DL, Wesley MJ, Porrino LJ (2011) Elevated gray and white matter densities in cocaine abstainers compared with current users. Psychopharmacology 218:681-692. CrossRef Medline

Jentsch JD, Taylor JR (1999) Impulsivity resulting from frontostriatal dysfunction in drug abuse: implications for the control of behavior by reward-related stimuli. Psychopharmacology 146:373-390. CrossRef Medline

Jentsch JD, Olausson P, De La Garza R 2nd, Taylor JR (2002) Impairments of reversal learning and response perseveration after repeated, intermittent cocaine administrations to monkeys. Neuropsychopharmacology 26: 183-190. CrossRef Medline

Krettek JE, Price JL (1977) The cortical projections of the mediodorsal nucleus and adjacent thalamic nuclei in the rat. J Comp Neurol 171:157-191. CrossRef Medline

Logothetis NK (2003) The underpinnings of the BOLD functional magnetic resonance imaging signal. J Neurosci 23:3963-3971. Medline

Madoz-Gúrpide A, Blasco-Fontecilla H, Baca-García E, Ochoa-Mangado E (2011) Executive dysfunction in chronic cocaine users: an exploratory study. Drug Alcohol Depend 117:55-58. CrossRef Medline

Mallet N, Pogosyan A, Márton LF, Bolam JP, Brown P, Magill PJ (2008a) Parkinsonian beta oscillations in the external globus pallidus and their relationship with subthalamic nucleus activity. J Neurosci 28:1424514258. CrossRef Medline

Mallet N, Pogosyan A, Sharott A, Csicsvari J, Bolam JP, Brown P, Magill PJ (2008b) Disrupted dopamine transmission and the emergence of exaggerated beta oscillations in subthalamic nucleus and cerebral cortex. J Neurosci 28:4795-4806. CrossRef Medline

Maroun M, Richter-Levin G (2003) Exposure to acute stress blocks the induction of long-term potentiation of the amygdala-prefrontal cortex pathway in vivo. J Neurosci 23:4406-4409. Medline

McAlonan K, Brown VJ (2003) Orbital prefrontal cortex mediates reversal learning and not attentional set shifting in the rat. Behav Brain Res 146: 97-103. CrossRef Medline

McDonald AJ (1987) Organization of amygdaloid projections to the mediodorsal thalamus and prefrontal cortex: a fluorescence retrograde transport study in the rat. J Comp Neurol 262:46-58. CrossRef Medline

McDonald AJ (1991) Organization of amygdaloid projections to the prefrontal cortex and associated striatum in the rat. Neuroscience 44:1-14. CrossRef Medline

Mcdonald AJ (1996) Glutamate and aspartate immunoreactive neurons of the rat basolateral amygdala: colocalization of excitatory amino acids and projections to the limbic circuit. J Comp Neurol 365:367-379. CrossRef Medline

Mcdonald AJ, Mascagni F, Guo L (1996) Projections of the medial and lateral prefrontal cortices to the amygdala: a Phaseolus vulgaris leucoagglutinin study in the rat. Neuroscience 71:55-75. CrossRef Medline

Mendelson JH, Mello NK (1996) Management of cocaine abuse and dependence. N Engl J Med 334:965-972. CrossRef Medline

Mitzdorf U (1985) Current source-density method and application in cat cerebral cortex: investigation of evoked potentials and EEG phenomena. Physiol Rev 65:37-100. Medline

Mogenson GJ, Jones DL, Yim CY (1980) From motivation to action: functional interface between the limbic system and the motor system. Prog Neurobiol 14:69-97. CrossRef Medline

Neymotin SA, Lee H, Park E, Fenton AA, Lytton WW (2011) Emergence of physiological oscillation frequencies in a computer model of neocortex. Front Comput Neurosci 5:19. CrossRef Medline

Noldy NE, Santos CV, Politzer N, Blair RD, Carlen PL (1994) Quantitative
EEG changes in cocaine withdrawal: evidence for long-term CNS effects. Neuropsychobiology 30:189-196. CrossRef Medline

Porrino LJ, Smith HR, Nader MA, Beveridge TJ (2007) The effects of cocaine: a shifting target over the course of addiction. Prog Neuropsychopharmacol Biol Psychiatry 31:1593-1600. CrossRef Medline

Porter JN, Olsen AS, Gurnsey K, Dugan BP, Jedema HP, Bradberry CW (2011) Chronic cocaine self-administration in rhesus monkeys: impact on associative learning, cognitive control, and working memory. J Neurosci 31:4926-4934. CrossRef Medline

Ragozzino ME (2002) The effects of dopamine $\mathrm{D}(1)$ receptor blockade in the prelimbic-infralimbic areas on behavioral flexibility. Learn Mem 9:18-28. CrossRef Medline

Ragozzino ME, Detrick S, Kesner RP (1999) Involvement of the prelimbicinfralimbic areas of the rodent prefrontal cortex in behavioral flexibility for place and response learning. J Neurosci 19:4585-4594. Medline

Robbins TW, Everitt BJ (1999) Drug addiction: bad habits add up. Nature 398:567-570. CrossRef Medline

Robinson OJ, Frank MJ, Sahakian BJ, Cools R (2010) Dissociable responses to punishment in distinct striatal regions during reversal learning. Neuroimage 51:1459-1467. CrossRef Medline

Rogers RD, Robbins TW (2001) Investigating the neurocognitive deficits associated with chronic drug misuse. Curr Opin Neurobiol 11:250-257. CrossRef Medline

Schnitzler A, Gross J (2005) Normal and pathological oscillatory communication in the brain. Nat Rev Neurosci 6:285-296. CrossRef Medline

Schoenbaum G, Setlow B (2003) Lesions of nucleus accumbens disrupt learning about aversive outcomes. J Neurosci 23:9833-9841. Medline

Schoenbaum G, Saddoris MP, Ramus SJ, Shaham Y, Setlow B (2004) Cocaine-experienced rats exhibit learning deficits in a task sensitive to orbitofrontal cortex lesions. Eur J Neurosci 19:1997-2002. CrossRef Medline

Schoenbaum G, Roesch MR, Stalnaker TA, Takahashi YK (2009) A new perspective on the role of the orbitofrontal cortex in adaptive behaviour. Nat Rev Neurosci 10:885-892. Medline

Soar K, Mason C, Potton A, Dawkins L (2012) Neuropsychological effects associated with recreational cocaine use. Psychopharmacology 222:633643. CrossRef Medline

Stalnaker TA, Roesch MR, Franz TM, Burke KA, Schoenbaum G (2006) Abnormal associative encoding in orbitofrontal neurons in cocaineexperienced rats during decision-making. Eur J Neurosci 24:2643-2653. CrossRef Medline

Stalnaker TA, Franz TM, Singh T, Schoenbaum G (2007a) Basolateral amygdala lesions abolish orbitofrontal-dependent reversal impairments. Neuron 54:51-58. CrossRef Medline

Stalnaker TA, Roesch MR, Franz TM, Calu DJ, Singh T, Schoenbaum G (2007b) Cocaine-induced decision-making deficits are mediated by miscoding in basolateral amygdala. Nat Neurosci 10:949-951. CrossRef Medline

Steriade M (2006) Grouping of brain rhythms in corticothalamic systems. Neuroscience 137:1087-1106. CrossRef Medline

Stern CE, Passingham RE (1995) The nucleus accumbens in monkeys (Macaca fascicularis). III. Reversal learning. Exp Brain Res 106:239-247. Medline

Tallon-Baudry C (2003) Oscillatory synchrony and human visual cognition. J Physiol Paris 97:355-363. CrossRef Medline

van Wingerden M, Vinck M, Lankelma JV, Pennartz CM (2010) Learningassociated gamma-band phase-locking of action-outcome selective neurons in orbitofrontal cortex. J Neurosci 30:10025-10038. CrossRef Medline

Volkow ND, Hitzemann R, Wang GJ, Fowler JS, Wolf AP, Dewey SL, Handlesman L (1992) Long-term frontal brain metabolic changes in cocaine abusers. Synapse 11:184-190. CrossRef Medline

Woicik PA, Moeller SJ, Alia-Klein N, Maloney T, Lukasik TM, Yeliosof O, Wang GJ, Volkow ND, Goldstein RZ (2009) The neuropsychology of cocaine addiction: recent cocaine use masks impairment. Neuropsychopharmacology 34:1112-1122. CrossRef Medline 